\title{
Topologie des fonctions régulières et cycles évanescents
}

\author{
Thomas BRÉLIVET \\ Laboratoire de Mathématiques Pures de Bordeaux \\ 351, cours de la Libération \\ 33405 Talence, France \\ Thomas.Brelivet@math.u-bordeaux.fr
}

Recibido: 21 de Enero de 2002

Aceptado: 9 de Julio de 2002

\begin{abstract}
One has two notions of vanishing cycles : the Deligne's general notion and a concrete one used recently in the study of polynomial functions. We compare these two notions which gives us in particular a relative connectivity result. We finish with an example of vanishing cycle calculation which shows the difficulty of a good choice of compactification.

\section{RÉSUMÉ}

On a deux notions de cycles évanescents : la notion générale introduite par Deligne et une autre plus concrète utilisée récemment pour l'étude des fonctions polynomiales. Nous comparons ces deux notions qui nous permettent d'avoir en particulier un résultat de connexité relative. Nous finissons par un exemple de calcul de cycles évanescents qui montre la difficulté du choix d'une bonne compactification.
\end{abstract}

2000 Mathematics Subject Classification: 14D05, 32S20, 32S60.

Key words: Applications régulières, cycles évanescents, connexité.

\section{Introduction}

Soit $f: X \longrightarrow S$ un morphisme non constant entre variétés algébriques complexes lisses $X$ de dimension $n$ et $S$ une courbe.

Pour le faisceau constant $\mathbb{C}_{X}$ et pour l'application $f$, on a les notions de cycles évanescents suivantes :

(i) la notion générale introduite par Deligne [6],

(ii) les définitions plus concrètes introduites par Neumann-Norbury [19] pour le cas affine $X=\mathbb{C}^{n}, S=\mathbb{C}$ et par Siersma-Tibăr [23] en général. 
Dans cette note nous commençons par expliquer la relation entre les définitions (i) et (ii). En faisant cela, nous dégageons une classe spéciale d'applications, les applications sympa (voir définition 2.4) qui jouissent de beaucoup de propriétés analogues au cas affine. Cette classe d'applications a aussi des propriétés intéressantes dans la théorie des D-modules, voir Dimca-Saito [9] et Sabbah [20], [21].

Dans la troisième partie, nous montrons certains résultats de connexité relative qui sont l'analogue faisceautique des résultats obtenus par Siersma-Tibăr [22], [23], [24], par Neumann-Norbury [19] et, plus récemment et dans un contexte faisceautique (faisceaux pervers sur $\mathbb{Z}$ ) par Hamm [14].

On obtient aussi des généralisations des théorèmes des cycles invariants locaux et globaux obtenus par Artal-Bartolo, Cassou-Noguès et Dimca [1], Neumann et Norbury [19], F. Michel et C. Weber [18], voir section 4.

La dernière section traitre de plusieurs exemples : singularités isolées ou de dimension 1 et aussi une compactification $\bar{f}: \bar{X} \longrightarrow S$ d'une certaine fonction $f$ dont le plongement $j: X \longrightarrow \bar{X}$ est un morphisme non affine.

\section{Comparaison de différentes notions de cycles évanescents}

Soit $f: X \rightarrow S$ un morphisme non constant entre variétés algébriques complexes lisses $X$ de dimension $n$ et $S$ une courbe. On sait qu'il existe un ensemble fini $B=$ $\left\{b_{1}, \ldots, b_{m}\right\}$ tel que

$$
f: X \backslash f^{-1}(B) \rightarrow S \backslash B
$$

soit une fibration topologique localement triviale. On choisit $B$ minimal ayant cette propriété ( $B$ est l'ensemble des valeurs de bifurcation). On note $F_{b}=f^{-1}(b)$ la fibre au dessus du point $b$ et $T\left(F_{b}\right)=f^{-1}\left(D_{\epsilon}(b)\right)$ le tube autour de la fibre $F_{b}, D_{\epsilon}(b)$ étant un disque fermé plongé dans $S$, de centre $b$ et suffisamment petit. Les fibres $F_{b_{i}}$, $i=1, \ldots, m$ sont dites fibres irrégulières et $F_{b^{\prime}}$ pour $b^{\prime} \notin B$ fibres régulières.

La définition naturelle suivante (en homologie et dans une situation similaire) a été introduite par D. Siersma et M. Tibăr dans [23].

Définition 2.1. Soit $b \in B, \epsilon>0$ suffisamment petit, $b^{\prime} \in D_{\epsilon}^{*}(b)=D_{\epsilon}(b) \backslash\{b\}$. On appelle $V^{q}(b)=H^{q+1}\left(T\left(F_{b}\right), F_{b^{\prime}}\right)^{1}$ les $q-(c o)$ cycles évanescents de l'application $f$ au point $b \in B$.

Remarque 2.1. La définition ne dépend pas du choix de $b^{\prime} \in D_{\epsilon}^{*}(b)$ ou de $\epsilon>0$.

Soit $b_{0} \in S \backslash \cup_{i=1}^{m} D_{\epsilon}\left(b_{i}\right)$ une valeur de référence pour $f$. On choisit des chemins $\gamma_{i}$ joignant $b_{0}$ à $D_{\epsilon}\left(b_{i}\right)$ tels que $\gamma_{i} \cap \gamma_{j}=b_{0}$ pour $i \neq j$. Soit $P$ l'union de ces chemins et $D$ l'union des disques autour des valeurs irrégulières de $f$. On note aussi $F=f^{-1}(P)$ qui se rétracte par déformation sur la fibre régulière $F_{b_{0}}$.

\footnotetext{
${ }^{1}$ Les groupes de cohomologie considérés ici sont à coefficients dans $\mathbb{Q}$ ou $\mathbb{C}$. Pour fixer les idées, on va travailler avec $\mathbb{C}$.
} 
Maintenant, on suppose $S=\mathbb{C}$. Comme $X$ se rétracte par déformation sur $f^{-1}(P \cup$ $D)$, on a alors par excision la proposition suivante, voir [4], [23], [19] pour le cas $X=\mathbb{C}^{n}$ et $[23]$ pour le cas général.

Proposition 2.1. Si $S=\mathbb{C}$, on a

(i)

$$
H^{q+1}(X, F)=\bigoplus_{b \in B} H^{q+1}\left(T\left(F_{b}\right), F_{b^{\prime}}\right)=\bigoplus_{b \in B} V^{q}(b)
$$

(ii)

$$
H^{q+1}\left(X, T\left(F_{b}\right)\right)=\bigoplus_{\substack{c \in B \\ c \neq b}} V^{q}(c)
$$

pour tout $b \in B$,

(iii) la suite exacte longue du triplet $\left(X, T\left(F_{b}\right), F_{b^{\prime}}\right)$ se décompose en suites exactes courtes de la forme

$$
0 \longrightarrow H^{q+1}\left(X, T\left(F_{b}\right)\right) \longrightarrow H^{q+1}\left(X, F_{b^{\prime}}\right) \longrightarrow V^{q}(b) \longrightarrow 0
$$

pour tout $b \in B$ et $b^{\prime} \in D_{\epsilon}(b)$.

On a la notion plus générale de cycles évanescents suivante, introduite par Deligne [6], que l'on va rappeler ici.

Soit $X$ un espace analytique complexe, on note $D^{b}(X)$ la catégorie dérivée de la catégorie des $\mathbb{C}_{X}$-modules $\bmod \left(\mathbb{C}_{X}\right)$ construite via les complexes de $\mathbb{C}_{X}$-modules bornés. On note aussi $D_{c}^{b}(X)$ la sous catégorie de $D^{b}(X)$ dont les complexes sont cohomologiquement constructibles (voir [3]).

Soit $X$ une variété algébrique (ou analytique) complexe, $f: X \rightarrow \mathbb{C}$ une fonction régulière (ou holomorphe) non constante.

Rappelons la définition des deux foncteurs

$$
\begin{aligned}
& D_{c}^{b}(X) \longrightarrow D_{c}^{b}\left(F_{0}\right) \text { et } D_{c}^{b}(X) \longrightarrow D_{c}^{b}\left(F_{0}\right) \\
& \mathcal{F}^{\bullet} \longmapsto \psi_{f}\left(\mathcal{F}^{\bullet}\right) \quad \mathcal{F}^{\bullet} \longmapsto \varphi_{f}\left(\mathcal{F}^{\bullet}\right)
\end{aligned}
$$

où $F_{0}=f^{-1}(0)$ est supposé non vide.

On a alors le diagramme

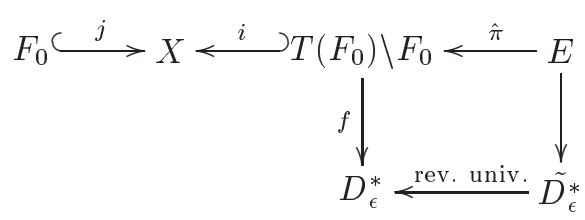

où $\epsilon$ a été choisi assez petit pour que $f: T\left(F_{0}\right) \backslash F_{0} \longrightarrow D_{\epsilon}^{*}$ soit une fibration topologique localement triviale (dans le cas analytique, cela est possible par exemple si $f$ 
est propre). Ici, $T\left(F_{0}\right)=f^{-1}\left(D_{\epsilon}\right)$ est le tube de la fibre $F_{0}$ et on regarde $E$ comme la fibre générale «universelle»de la fibration $f: T\left(F_{0}\right) \backslash F_{0} \longrightarrow D_{\epsilon}^{*}$ (le carré à droite étant cartésien).

Définition 2.2. (Cycles proches) On note $\psi_{f}$ le foncteur des cycles proches relatif $\grave{a}$ la fonction $f$ le foncteur de $D_{c}^{b}(X)$ dans $D_{c}^{b}\left(F_{0}\right)$ qui a un complexe $\mathcal{F}^{\bullet}$ associe le complexe $\psi_{f} \mathcal{F}^{\bullet}=j^{-1} R(i \circ \hat{\pi})_{*}(i \circ \hat{\pi})^{-1}\left(\mathcal{F}^{\bullet}\right)$.

Définition 2.3. (Cycles évanescents) On note $\varphi_{f}$ le foncteur des cycles évanescents relatif à la fonction $f$ et can $: \psi_{f}\left(\mathcal{F}^{\bullet}\right) \longrightarrow \varphi_{f}\left(\mathcal{F}^{\bullet}\right)$ le morphisme canonique défini par le triangle distingué

$$
j^{-1} \mathcal{F}^{\bullet} \stackrel{c}{\longrightarrow} \psi_{f}\left(\mathcal{F}^{\bullet}\right) \stackrel{\operatorname{can}}{\longrightarrow} \varphi_{f}\left(\mathcal{F}^{\bullet}\right) \stackrel{[+1]}{\longrightarrow} .
$$

\section{Remarque 2.2.}

- On peut aussi définir les cycles proches et les cycles évanescents pour $f: T \rightarrow D$ où $T$ est un espace complexe, $D$ un disque dans $\mathbb{C}$ de centre $0, f$ une application continue telle que $f_{\mid T \backslash f^{-1}(0)} \rightarrow D^{*}$ soit une fibration topologique localement triviale et $\mathcal{F}_{\mid T \backslash f^{-1}(0)}$ un complexe de faisceaux cohomologiquement constructibles.

- Les foncteurs $\psi_{f}[-1]$ et $\varphi_{f}[-1]$ sont des foncteurs t-exacts, c'est à dire que l'image par l'un de ces deux foncteurs d'un faisceau pervers est un faisceau pervers (voir [15], Corollary 10.3.13).

On veut comparer les cycles évanescent de la définition 2.1 avec ceux de la définition 2.3. On sait que le foncteur cycles évanescents commute avec les foncteurs images directes par les applications propres. C'est pour cela qu'il est naturel de considérer des compactifications de l'application $f$.

Soit $\bar{f}: \bar{X} \rightarrow S$ une compactification de $f: X \rightarrow S$, c'est-à-dire $: \bar{X}$ est une variété algébrique et $\bar{f}$ un prolongement de $f$ qui est propre. On note $j: X \rightarrow \bar{X}$ l'inclusion naturelle.

Lemme 2.1. Pour tout complexe $\mathcal{G}^{\bullet} \in D_{c}^{b}(\bar{X})$, tout $b \in B$ et tout $b^{\prime} \in D_{\epsilon}(b) \backslash\{b\}$ on a l'égalité suivante

$$
\mathbb{H}^{q+1}\left(T\left(\bar{F}_{b}\right), \bar{F}_{b^{\prime}}, \mathcal{G}^{\bullet}\right)=\mathbb{H}^{q}\left(\bar{F}_{b}, \varphi_{t_{b} \circ \bar{f}} \mathcal{G}^{\bullet}\right)
$$

où t $t_{b}$ est une coordonnée locale de $S$ en $b$.

Démonstration. Il suffit d'utiliser la formule de changement de base ([15], p. 358)

$$
\varphi_{t_{b}}\left(R \bar{f}_{*} \mathcal{G}^{\bullet}\right)=R \Gamma\left(\varphi_{t_{b} \circ \bar{f}}\left(\mathcal{G}^{\bullet}\right)\right)
$$

et d'utiliser le fait que l'on a $\varphi_{t_{b}}^{q}\left(R \bar{f}_{*} \mathcal{G} \bullet\right)=\mathbb{H}^{q+1}\left(T\left(\bar{F}_{b}\right), \bar{F}_{b^{\prime}}, \mathcal{G}^{\bullet}\right)$.

Proposition 2.2. On $a V^{q}(b)=\mathbb{H}^{q}\left(\bar{F}_{b}, \varphi_{t_{b}} \circ \bar{f} j_{*} \mathbb{Q}_{X}\right)=\varphi_{t_{b}}^{q}\left(R f_{*} \mathbb{Q}_{X}\right)$. 
Démonstration. En appliquant le lemme précédent à $\mathcal{G}^{\bullet}=R j_{*} \mathbb{Q}_{X}$, on obtient

$$
\mathbb{H}^{q}\left(\bar{F}_{b}, \varphi_{t_{b} \circ \bar{f}} R j_{*} \mathbb{Q}_{X}\right)=\varphi_{t_{b}}^{q}\left(R f_{*} \mathbb{Q}_{X}\right) .
$$

Considérons le diagramme commutatif suivant

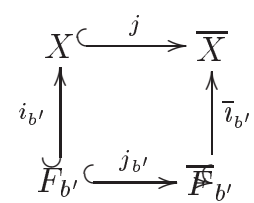

où $i_{b^{\prime}}$ est l'inclusion de $F_{b^{\prime}}$ dans $X, \bar{\imath}_{b^{\prime}}$ l'inclusion de $\bar{F}_{b^{\prime}}$ dans $\bar{X}$ et $j_{b^{\prime}}$ l'inclusion de $F_{b^{\prime}}$ dans $\bar{F}_{b^{\prime}}$.

En adaptant la preuve de Sabbah de la proposition 8.3 dans [21] au cas d'une fibre régulière, on montre que

$$
\bar{\imath}_{b^{\prime}}^{-1}\left(R j_{*} \mathbb{Q}_{X}\right)=R j_{b^{\prime} *}\left(i_{b^{\prime}}^{-1} \mathbb{Q}_{X}\right)
$$

On a le même type de propriétés avec $T\left(F_{b}\right)$ au lieu de $F_{b^{\prime}}$ en appliquant directement les définitions (et en se ramenant à un tube ouvert). Cela nous permet de voir que

$$
V^{q}(b)=\mathbb{H}^{q+1}\left(T\left(\bar{F}_{b}\right), \bar{F}_{b^{\prime}}, R j_{*} \mathbb{Q}_{X}\right) .
$$

Ceci nous amène naturellement à poser les notations suivantes.

Notations 2.1. On pose

et

$$
\begin{gathered}
\Sigma_{b}^{q}=\operatorname{supp} \varphi_{t_{b} \circ \bar{f}}^{q}\left(R j_{*} \mathbb{C}_{X}\right), \\
\Sigma_{b}=\cup_{q} \Sigma_{b}^{q},
\end{gathered}
$$

$$
d(\bar{f})=\max _{b} \operatorname{dim} \Sigma_{b} .
$$

Si on dispose d'une stratification de Whitney $\mathcal{S}$ de $\bar{X}$ telle que $X$ soit une strate et si on pose $d(\bar{f}, \mathcal{S})=\operatorname{dim} \operatorname{Sing}_{\mathcal{S}}(\bar{f})$, alors on a

$$
d(\bar{f}) \leq d(\bar{f}, \mathcal{S})
$$

Considérons les cycles évanescents associés aux faisceaux constructibles $\mathcal{F}^{q}=$ $R^{q} f_{*}\left(\mathbb{C}_{X}\right)$ où $q$ est un entier positif.

Pour $q$ un entier positif, soit $j_{b}^{q}: H^{q}\left(T\left(F_{b}\right)\right) \longrightarrow H^{q}\left(F_{b^{\prime}}\right)$ le morphisme induit par l'inclusion $F_{b^{\prime}} \longrightarrow T\left(F_{b}\right)$. Dans le triangle

$$
\mathcal{F}_{b}^{q} \stackrel{c}{\longrightarrow} \psi_{t_{b}}\left(\mathcal{F}^{q}\right) \longrightarrow \varphi_{t_{b}}\left(\mathcal{F}^{q}\right) \stackrel{+1}{\longrightarrow}
$$


on a $\mathcal{F}_{b}^{q}=H^{q}\left(T\left(F_{b}\right)\right), \psi_{t_{b}}\left(\mathcal{F}^{q}\right)=\mathcal{F}_{b^{\prime}}^{q}=H^{q}\left(F_{b^{\prime}}\right)$ et le morphisme de comparaison $c$ coïncide avec $j_{b}^{q}$. Cela nous donne

$$
\varphi_{t_{b}}^{-1}\left(\mathcal{F}^{q}\right)=\operatorname{Ker} j_{b}^{q}, \quad \varphi_{t_{b}}^{0}\left(\mathcal{F}^{q}\right)=\operatorname{Coker} j_{b}^{q} .
$$

En comparant la suite exacte longue associée au triangle (1) et la suite exacte longue de cohomologie associée au couple $\left(T\left(F_{b}\right), F_{b^{\prime}}\right)$, on obtient

$$
0 \longrightarrow \varphi_{t_{b}}^{0}\left(\mathcal{F}^{q}\right) \longrightarrow \varphi_{t_{b}}^{q}\left(R f_{*} \mathbb{C}_{X}\right)=V^{q}(b) \longrightarrow \varphi_{t_{b}}^{-1}\left(\mathcal{F}^{q+1}\right) \longrightarrow 0 .
$$

On a le résultat suivant

Proposition 2.3 Soient $f: X \longrightarrow S$ un morphisme non constant entre variétés algébriques complexes lisses $X$ de dimension $n$ et $S$ une courbe. Soient $q$ un entier positif, $\mathcal{F}^{\bullet}=R^{q} f_{*} \mathbb{C}_{X}$ et $j_{b}^{q}: H^{q}\left(T\left(F_{b}\right)\right) \longrightarrow H^{q}\left(F_{b^{\prime}}\right)$ le morphisme induit par l'inclusion d'une fibre générale dans un tube autour de $F_{b}=f^{-1}(b)$, où b est une valeur de bifurcation. Soit $q$ un entier positif donné alors les affirmations suivantes sont équivalentes

(i) $j_{b}^{q}$ est injectif pour toute valeur de bifurcation $b$,

(ii) le faisceau $\mathcal{F}^{q}$ n'a pas de section à support fini,

(iii) le faisceau $\mathcal{F}^{q}[1]$ est pervers,

(iv) $\mathcal{F}^{q}[1]={ }^{p} R^{q+1} f_{*} \mathbb{C}_{X}$ est dans $D_{c}^{b}(X)$.

Démonstration. L'équivalence (i) $\Leftrightarrow$ (ii) et l'implication (iv) $\Rightarrow$ (iii) sont immédiates.

Les autres implications résultent des deux faits suivants bien connus sur les faisceaux pervers (pour une preuve, on peut se rapporter à [8]).

Lemme 2.2. Soit $\mathcal{F}^{\bullet} \in D_{c}^{b}(S)$ où $S$ est une courbe complexe lisse. Alors on a des suites exactes courtes

$$
0 \longrightarrow \mathcal{H}^{0}\left({ }^{p} \mathcal{H}^{k}\left(\mathcal{F}^{\bullet}\right)\right) \longrightarrow \mathcal{H}^{k}\left(\mathcal{F}^{\bullet}\right) \longrightarrow \mathcal{H}^{-1}\left({ }^{p} \mathcal{H}^{k+1}\left(\mathcal{F}^{\bullet}\right)\right) \longrightarrow 0
$$

pour tout entier $k$.

Lemme 2.3. Soit $\mathcal{F}^{\bullet} \in \operatorname{Perv}(S)$ où $S$ est une courbe complexe lisse. On a un isomorphisme $\mathcal{F}^{\bullet}=\mathcal{H}^{-1}\left(\mathcal{F}^{\bullet}\right)[1]$ si et seulement si une des deux conditions équivalentes suivantes est remplie.

(i) $\mathcal{H}^{0}\left(\mathcal{F}^{\bullet}\right)=0$,

(ii) pour tout point $a \in S$ le morphisme canonique can $: \psi_{t_{a}}\left(\mathcal{F}^{\bullet}\right) \longrightarrow \varphi_{t_{a}}\left(\mathcal{F}^{\bullet}\right)$ est surjectif, $t_{a}$ étant une coordonnée locale en a.

Exemple 2.1. Ces conditions sont remplies pour $S=\mathbb{C}, X$ contractile voir ci-dessous et aussi lorsque $X$ est affine et $\Sigma_{b} \subset X$ pour tout $b$, voir Sabbah [21], où ces fonctions sont appelées «cohomologiquement modérées». La remarque 5.4 montre que ces conditions sont aussi remplies pour d'autres classes d'exemples. 
Par contre elles ne sont pas remplies pour $X=\mathbb{C}^{*}, f: \mathbb{C}^{*} \longrightarrow \mathbb{C}$ l'inclusion. En effet, le morphisme $j_{0}^{1}: H^{1}$ (disque épointé) $\longrightarrow H^{1}$ (point) n'est pas injectif.

Définition 2.4. On dit que l'application $f: X \longrightarrow S$ est sympa si elle vérifie les conditions (i)-(iv) de la proposition 2.3 pour tout $q$.

Remarque 2.3. Dans le cas d'une application régulière $f: X \rightarrow \mathbb{C}$ avec $X$ contractile, la suite de Mayer-Vietoris nous dit que l'on a la suite exacte (voir [19])

$$
0 \longrightarrow H^{q}\left(F_{b_{0}}\right) \oplus \bigoplus_{i=1}^{m} H^{q}\left(T\left(F_{b_{i}}\right)\right) \longrightarrow \bigoplus_{i=1}^{m} H^{q}\left(F_{b_{i}^{\prime}}\right) \longrightarrow 0 \text {, pour } q>0
$$

Donc on a une injection $H^{q}\left(T\left(F_{b_{i}}\right)\right) \hookrightarrow H^{q}\left(F_{b_{i}^{\prime}}\right)$ qui nous dit que $V^{q}(b)=$ Coker $\left(H^{q}\left(T\left(F_{b}\right)\right) \rightarrow H^{q}\left(F_{b^{\prime}}\right)\right)$. Dans le cas $X=\mathbb{C}^{n}$ ceci est la notion de cocycles évanescents introduite par Neumann-Norbury [19]. On a de plus $\tilde{H}^{q}(F)=\bigoplus_{i=1}^{m} V^{q}\left(b_{i}\right)$ pour tout $q$.

Remarque 2.4. On sait qu'un germe de faisceaux pervers $\mathcal{F}^{\bullet}$ à l'origine de $\mathbb{C}$ est déterminé à isomorphisme près par le diagramme

$$
\psi_{t}\left(\mathcal{F}^{\bullet}\right) \underset{\operatorname{var}}{\stackrel{\operatorname{can}}{\rightleftarrows}} \varphi_{t}\left(\mathcal{F}^{\bullet}\right)
$$

Dans le cas d'une application sympa $f: X \longrightarrow S$ le germe du faisceau $\mathcal{F}^{q}[1]$ au point $b \in S$ est déterminé par le diagramme

$$
H^{q}\left(F_{b^{\prime}}\right) \underset{\text { var }}{\stackrel{\delta}{\rightleftarrows}} H^{q+1}\left(T\left(F_{b}\right), F_{b^{\prime}}\right)=V^{q}(c)
$$

où var est le dual du morphisme en homologie

$$
H_{q}\left(F_{b^{\prime}}\right) \simeq H_{q+1}\left(F_{b^{\prime}} \times\left(S^{1}, b^{\prime}\right)\right)=H_{q+1}\left(f^{-1}\left(\partial D_{\epsilon}(b)\right), F_{b^{\prime}}\right) \stackrel{i_{*}}{\longrightarrow} H_{q+1}\left(T\left(F_{b}\right), F_{b^{\prime}}\right)
$$

où $S^{1}=\partial D_{\epsilon}(b)$, voir [23] pour une construction similaire, $T\left(F_{b}\right)$ étant remplacé par $X$ chez eux.

On obtient pour le cas $S=\mathbb{C}$ (en utilisant les chemins $\gamma_{i}$ ) deux opérateurs de monodromie

$$
m_{b}^{q}: H^{q}(F) \longrightarrow H^{q}(F), \quad m_{b}^{q}=1+\operatorname{var} \circ \delta
$$

et

$$
m_{b, \text { rel }}^{q}: H^{q+1}(X, F) \longrightarrow H^{q+1}(X, F), \quad m_{b, \text { rel }}^{q}=1+\delta \circ \operatorname{var} \circ j^{q}
$$

avec $j_{b}^{q}: H^{q+1}(X, F) \longrightarrow H^{q+1}\left(T\left(F_{b}\right), F\right)$.

Cela induit des représentations de monodromie

$$
\rho^{q}: \pi_{1}\left(\mathbb{C} \backslash B, b_{0}\right) \longrightarrow \operatorname{Aut}\left(H^{q}(F)\right),
$$




$$
\rho_{\mathrm{rel}}^{q}: \pi_{1}\left(\mathbb{C} \backslash B, b_{0}\right) \longrightarrow \operatorname{Aut}\left(H^{q+1}(X, F)\right) .
$$

Remarque 2.5. On peut aussi définir deux opérateurs de monodromie à l'infini $m_{\infty}^{q}$ et $m_{\infty, \text { rel }}^{q}$ qui correspondent respectivement à $\rho^{q}(\gamma)$ et à $\rho_{\text {rel }}^{q}(\gamma)$ pour $\gamma$ un grand lacet dans $\mathbb{C}$ bord d'un disque qui contient $B$ dans son intérieur. On peut montrer comme dans [11] que $\rho_{\text {rel }}^{q}$ est triviale si et seulement si $m_{\infty, \text { rel }}^{q}=I d$.

Pour une fonction cohomologiquement modérée, Sabbah a obtenu des résultats très intéressants sur l'opérateur de monodromie à l'infini $m_{\infty, \text {,rel }}^{n-1}$, voir [21] remarque (10.3).

Question ouverte. Existe-t'il une application $f: X \longrightarrow \mathbb{C}$ telle que la représentation $\rho_{\text {rel }}^{q}$ soit triviale mais pas la représentation $\rho^{q}$ ?

\section{Annulations de cycles évanescents et connexité relative}

La suite exacte (2) et la proposition 2.3 nous donnent le corollaire suivant.

Corollaire 3.1. Si $V^{q}(b)=0$ pour tout $q \leq q_{0}$ et tout b, alors les faisceaux $R^{q} f_{*} \mathbb{C}_{X}={ }^{p} R^{q+1} f_{*} \mathbb{C}_{X}[-1]$ sont localement constants pour $q \leq q_{0}$ et $R^{q_{0}+1} f_{*} \mathbb{C}_{X}=$ ${ }^{p} R^{q_{0}+2} f_{*} \mathbb{C}_{X}[-1]$.

En effet, pour $q \leq q_{0}$ on a un isomorphisme

$$
R^{q} f_{*} \mathbb{C}_{X}=\widetilde{H^{q}(F)}
$$

où $\widetilde{M}$ est le système local constant associé à un $\mathbb{C}$-espace vectoriel $M$.

Dans la section précédente, on a vu que $V^{q}(b)=\mathbb{H}^{q}\left(\bar{f}^{-1}(b), \varphi_{t_{b} \circ \bar{f}} R j_{*} \mathbb{C}_{X}\right)$. Ce résultat va nous permettre d'avoir un théorème d'annulation pour les cycles évanescents.

Proposition 3.1. Supposons que $j$ soit affine.

Alors, on a

$$
V^{q}(b)=0 \text { pour } q \leq n-d(\bar{f})-2 \text { et pour } q \geq n+d(\bar{f}) .
$$

Démonstration. Le faisceau $\mathbb{C}_{X}[n]$ est un faisceau pervers (voir [17]) et $R j_{*}$ est un foncteur t-exact donc $R j_{*} \mathbb{C}_{X}[n] \in \operatorname{Perv}(\bar{X})$. On a aussi

$$
V^{q}(b)=\mathbb{H}^{q+1-n}\left(\Sigma_{b},{ }^{p} \varphi_{t_{b} \circ \bar{f}} R j_{*} \mathbb{C}_{X}[n]\right) .
$$

Ces deux résultats avec le théorème d'Artin (voir [15], Proposition 10.3.17) impliquent que $V^{q}(b)=0$ pour $q+1-n \leq-d(\bar{f})-1$ et pour $q+1-n \geq d(\bar{f})+1$.

Lorsque $S=\mathbb{C}$, on a $H^{q+1}(X, F)=\bigoplus_{b} V^{q}(b)$, donc la proposition précédente nous dit que $H^{q+1}(X, F)=0$ pour $q \leq n-d(\bar{f})-2$ et pour $q \geq n+d(\bar{f})$. Si de plus 
$X=\mathbb{C}^{n}$ ou $X$ contractile alors on a $\tilde{H}^{q}(F)=0$ pour $0 \leq q \leq n-d(\bar{f})-2$ et pour $q \geq n$. Ce dernier résultat est clair lorsque $X$ est affine, car alors $F$ est aussi affine.

L'exemple 2.1 montre que le faisceau $R^{n} f_{*} \mathbb{C}_{X}[1]$ n'est pas en général pervers même lorsque $d(\bar{f})=0$.

Un résultat de C. Sabbah [20] qui généralise un résultat de A. Dimca et M. Saito [9] nous permet d'obtenir le corollaire suivant

Corollaire 3.2. Soient $X$ une variété affine lisse et $f: X \longrightarrow \mathbb{C}$ une application régulière non constante. Alors le complexe

$$
K^{\bullet}: 0 \longrightarrow \Omega^{0}(X) \stackrel{D_{f}}{\longrightarrow} \Omega^{1}(X) \stackrel{D_{f}}{\longrightarrow} \cdots \stackrel{D_{f}}{\longrightarrow} \Omega^{n}(X) \longrightarrow 0
$$

où $D_{f}(\omega)=d \omega-d f \wedge \omega$ vérifie $H_{0}^{q}\left(K^{\bullet}\right)=0$ pour $q_{0} \leq n-d(\bar{f})-2$, où l'entier $d(\bar{f})$ est calculé par rapport à une compactification $X \subset \bar{X}$ quelconque.

La proposition suivante nous donne une annulation des groupes de cohomologie à support compact dans le cas où $f: X \rightarrow \mathbb{C}$ est une application sympa.

Proposition 3.2. Si $\tilde{b}_{q}(F)=0$ pour $0 \leq q \leq q_{0}$ alors $H_{c}^{q}\left(F_{b}\right)=0$ pour $2 n-q_{0}-1 \leq$ $q \leq 2 n-3$ et tout $b \in \mathbb{C}$.

En particulier si $F_{b}$ est lisse et connexe $H_{c}^{q}\left(F_{b}\right) \simeq H^{2 n-2-q}\left(F_{b}\right)$ d'où

$$
H^{0}\left(F_{b}\right)=\mathbb{C}, \quad H^{1}\left(F_{b}\right)=0, \ldots, \quad H^{q_{0}-1}\left(F_{b}\right)=0 .
$$

Démonstration. On dispose de la suite exacte pour la cohomologie à support compact

$$
\begin{aligned}
\cdots \longrightarrow H_{c}^{q-1}\left(F_{b}\right) & \longrightarrow H_{c}^{q}\left(T\left(F_{b}\right) \backslash F_{b}\right) \longrightarrow H_{c}^{q}\left(T\left(F_{b}\right)\right) \longrightarrow \\
& \longrightarrow H_{c}^{q}\left(F_{b}\right) \longrightarrow H_{c}^{q+1}\left(T\left(F_{b}\right) \backslash F_{b}\right) \longrightarrow \cdots
\end{aligned}
$$

Par dualité, on a $H_{c}^{q}\left(T\left(F_{b}\right)\right)=H^{2 n-q}\left(T\left(F_{b}\right)\right)^{\vee}$ or ce dernier groupe est nul pour $1 \leq 2 n-q \leq q_{0}$ puisque l'on a une injection de $H^{q}\left(T\left(F_{b}\right)\right)$ dans $H^{q}(F)$.

Maintenant, la suite exacte de Wang

$$
\begin{aligned}
\cdots \longrightarrow H^{2 n-q-1}(F) \longrightarrow H_{c}^{q}\left(T\left(F_{b}\right) \backslash F_{b}\right) & =H^{2 n-q}\left(T\left(F_{b}\right) \backslash F_{b}\right) \longrightarrow \\
\longrightarrow & \longrightarrow H^{2 n-q}(F) \longrightarrow H^{2 n-q}(F) \longrightarrow \cdots
\end{aligned}
$$

nous donne le résultat pour $2 n-q_{0} \leq q \leq 2 n-3$. Pour l'exposant $2 n-q_{0}-1$ on considère la première suite exacte et on remarque que la flèche $H_{c}^{q}\left(T\left(F_{b}\right) \backslash F_{b}\right) \longrightarrow$ $H_{c}^{q}\left(T\left(F_{b}\right)\right)$ est surjective. Cette dernière est surjective car par dualité elle correspond à $H^{q}\left(T\left(F_{b}\right)\right) \rightarrow H^{q}\left(T\left(F_{b}\right) \backslash F_{b}\right)$ qui factorise l'injection $H^{q}\left(T\left(F_{b}\right)\right) \rightarrow H^{q}(F)$.

Théorème 3.1. Soient $f: X \rightarrow \mathbb{C}$ une application régulière où $X$ est une variété complexe lisse de dimension $n$ et $\bar{f}: \bar{X} \rightarrow \mathbb{C}$ une compactification telle que l'injection naturelle $j: X \rightarrow \bar{X}$ soit affine. 
Alors

$$
\begin{array}{ccccc}
b_{q+1}(X, F)=0 & \text { si } & q \leq n-d(\bar{f})-2 & \text { ou } & q \geq n+d(\bar{f}), \\
b_{q+1}\left(X, F_{b}\right)=0 & \text { si } \quad q \leq n-d(\bar{f})-3 \quad \text { ou } & q \geq n+d(\bar{f})+1 .
\end{array}
$$

Si de plus

$$
\Sigma_{b}^{\mathrm{f}} \text { fermé dans } X \text { et } \operatorname{dim}\left(\Sigma_{b}^{\infty}\right) \leq d(\bar{f})-1,
$$

alors

$$
b_{q+1}\left(X, F_{b}\right)=0 \quad \text { si } \quad q \leq n-d(\bar{f})-2 \quad \text { ou } \quad q \geq n+d(\bar{f}) .
$$

où $\Sigma_{b}^{\mathrm{f}}=\Sigma_{b} \cap X, \Sigma_{b}^{\infty}=\Sigma_{b} \cap X_{\infty}$ et $X_{\infty}=\bar{X} \backslash X$.

Le résultat homotopique analogue pour $\bar{d}(f)=0$ est démontré dans [23].

Démonstration. Notre preuve suit dans les grandes lignes une preuve de l'article de Hamm [14], où il démontre une version plus précise du théorème 3.1, pour le cas $d(\bar{f})=0$. Voir aussi [23] pour le cas $\bar{d}(f)=0$.

D'après la proposition 2.1 on a $H^{q+1}(X, F)=\oplus_{i=1}^{m} V^{q}\left(b_{i}\right)$. La proposition 3.1 nous dit que $V^{q}\left(b_{i}\right)=0$ pour $q \leq n-d(\bar{f})-2, q \geq n+d(\bar{f})$ et pour tout $i$, d'où la première affirmation.

Les faisceaux $R^{q} f_{*} \mathbb{C}_{X}$ sont localement constants pour $q \leq n-d(\bar{f})-2$, voir le corollaire 3.1 .

Maintenant on introduit les notations suivantes : $f_{b}=f_{\mid F_{b}}, \bar{f}_{b}=\bar{f}_{\mid \bar{F}_{b}}$ et les inclusions suivantes

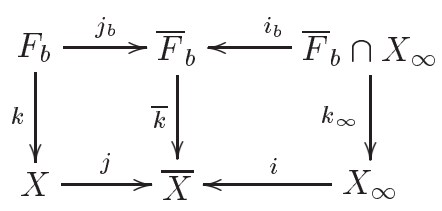

Il suffit de montrer que le morphisme naturel

$$
H^{q}\left(T\left(F_{b}\right), \mathbb{C}\right) \longrightarrow H^{q}\left(F_{b}, \mathbb{C}\right)
$$

induit par l'inclusion de la fibre $F_{b}$ dans son tube $T\left(F_{b}\right)$ est

(i) un isomorphisme si $q \leq n-d(\bar{f})-3$ et $q \geq n+d(\bar{f})+2$,

(ii) un épimorphisme si $q=n+d(\bar{f})+1$,

et si de plus $f$ vérifie la condition supplémentaire alors c'est

(i) un isomorphisme si $q \leq n-d(\bar{f})-2$ et $q \geq n+d(\bar{f})+1$,

(ii) un épimorphisme si $q=n+d(\bar{f})$. 
En effet, il suffit d'écrire les suites exactes de $(X, F),\left(X, T\left(F_{b}\right)\right),\left(X, F_{b}\right)$, pour $q$ petit et $\left(X, T\left(F_{b}\right), F_{b}\right)$ ainsi que la proposition 2.1 (iii) pour $q$ grand. On a les isomorphismes suivants

$$
\begin{aligned}
H^{q}\left(T\left(F_{b}\right), \mathbb{C}\right) & =R^{q} f_{*}\left(\mathbb{C}_{X}\right)_{b}=\mathbb{R}^{q} \bar{f}_{*}\left(R j_{*} \mathbb{C}_{X}\right)_{b} \\
& =\mathbb{H}^{q}\left(\overline{F_{b}}, \bar{k}^{-1} R j_{*} \mathbb{C}_{X}\right)=\mathbb{R}^{q} \bar{f}_{b *}\left(\bar{k}^{-1} R j_{*} \mathbb{C}_{X}\right)
\end{aligned}
$$

(le deuxième provient de $R f_{*}=R \bar{f}_{*} \circ R j_{*}$ et le troisième du fait que $\bar{f}$ est propre).

D'autre part on a les isomorphismes suivants

$$
\begin{aligned}
H^{q}\left(F_{b}, \mathbb{C}\right) & =H^{q}\left(F_{b}, k^{-1} \mathbb{C}_{X}\right)=\mathbb{H}^{q}\left(\bar{F}_{b}, R j_{b *} k^{-1} \mathbb{C}_{X}\right) \\
& =\mathbb{R}^{q} \bar{f}_{b *}\left(R j_{b *} k^{-1} \mathbb{C}_{X}\right)=\mathbb{R}^{q} \bar{f}_{b *}\left(R j_{b *} j_{b}^{-1} \bar{k}^{-1} R j_{*} \mathbb{C}_{X}\right)
\end{aligned}
$$

où le dernier isomorphisme vient de

$$
j_{b}^{-1} \bar{k}^{-1} R j_{*} \mathbb{C}_{X}=k^{-1} j^{-1} R j_{*} \mathbb{C}_{X}=k^{-1} \mathbb{C}_{X} .
$$

Donc le morphisme $(3)$ induit par l'inclusion $F_{b} \longrightarrow T\left(F_{b}\right)$ peut être remplacé par un morphisme

$$
\mathbb{R}^{q} \bar{f}_{b *}\left(\mathcal{F}^{\bullet}\right) \longrightarrow \mathbb{R}^{q} \bar{f}_{b *}\left(R j_{b *} j_{b}^{-1} \mathcal{F}^{\bullet}\right)
$$

avec $\mathcal{F}^{\bullet}=\bar{k}^{-1} R j_{*} \mathbb{C}_{X}$ qui provient en effet du morphisme d'adjonction $\mathcal{F}^{\bullet} \longrightarrow$ $R j_{b *} j_{b}^{-1} \mathcal{F}^{\bullet}$ par l'application du foncteur $\mathbb{R}^{q_{0}} \bar{f}_{b *}$.

Nous considérons maintenant le triangle distingué d'adjonction suivant

$$
i_{b !} i_{b}^{!} \mathcal{F}^{\bullet} \longrightarrow \mathcal{F}^{\bullet} \longrightarrow R j_{b *} j_{b}^{-1} \mathcal{F}^{\bullet} \stackrel{+1}{\longrightarrow}
$$

Soit $\mathcal{G}^{\bullet}=i_{b !} i_{b}^{!} \mathcal{F}^{\bullet}$. Alors on a une suite exacte longue

$$
\cdots \longrightarrow \mathbb{R}^{q} \bar{f}_{b *} \mathcal{G}^{\bullet} \longrightarrow \mathbb{R}^{q} \bar{f}_{b *} \mathcal{F}^{\bullet} \longrightarrow \mathbb{R}^{q} \bar{f}_{b *} R j_{b *} j_{b}^{-1} \mathcal{F}^{\bullet} \longrightarrow \mathbb{R}^{q+1} \bar{f}_{b *} \mathcal{G}^{\bullet} \longrightarrow \cdots
$$

et il suffit de montrer que $\mathbb{R}^{q} \bar{f}_{b *} \mathcal{G} \bullet=0$ pour $q \leq n-d(\bar{f})-2, q \geq n+d(\bar{f})+2$ et aussi pour $q=n-d(\bar{f})-1, q=n+d(\bar{f})$ si $f$ vérifie la condition supplémentaire.

On a deux triangles distingués dans $D_{c}^{b}\left(\bar{F}_{b}\right)$

$$
{ }^{p} \psi_{\bar{f}-b} R j_{*} \mathbb{C}_{X}[n] \longrightarrow{ }^{p} \varphi_{\bar{f}-b} R j_{*} \mathbb{C}_{X}[n] \longrightarrow \bar{k}^{-1} R j_{*} \mathbb{C}_{X}[n] \stackrel{+1}{\longrightarrow}
$$

et

$$
\bar{k}^{!} R j_{*} \mathbb{C}_{X}[n] \longrightarrow{ }^{p} \varphi_{\bar{f}-b} R j_{*} \mathbb{C}_{X}[n] \longrightarrow{ }^{p} \psi_{\bar{f}-b} R j_{*} \mathbb{C}_{X}[n] \stackrel{+1}{\longrightarrow} .
$$

On applique le $\delta$-foncteur $i_{b}^{!}$au triangle (5) en remarquant que $i_{b}^{!} \frac{{ }^{!}}{!} R j_{*}=k_{\infty}^{!} R j_{*}=0$. 
Donc le triangle (5) nous donne un quasi-isomorphisme

$$
i_{b}^{! p} \varphi_{\bar{f}-b} R j_{*} \mathbb{C}_{X}[n] \longrightarrow i_{b}^{p} \psi_{\bar{f}-b} R j_{*} \mathbb{C}_{X}[n] .
$$

Maintenant, on applique le $\delta$-foncteur $R \bar{f}_{b *} i_{b !} i_{b}^{!}$au triangle (4) et on obtient

$$
\cdots \rightarrow \mathbb{H}^{q}\left(\Sigma_{b}^{\infty}, i_{b}^{!} \mathcal{B}^{\bullet}\right) \rightarrow \mathbb{H}^{q}\left(\Sigma_{b}^{\infty}, i_{b}^{!} \mathcal{B}^{\bullet}\right) \rightarrow \mathbb{R}^{q+n} \bar{f}_{b} \mathcal{G}^{\bullet} \rightarrow \mathbb{H}^{q+1}\left(\Sigma_{b}^{\infty}, i_{b}^{!} \mathcal{B}^{\bullet}\right) \rightarrow \cdots
$$

où $\mathcal{B}^{\bullet}={ }^{p} \varphi_{\bar{f}-b} R j_{*} \mathbb{C}_{X}[n]$.

On a le triangle d'attachement

$$
i_{b !} i_{b}^{!} \mathcal{B}^{\bullet} \longrightarrow \mathcal{B}^{\bullet} \longrightarrow R j_{b *} j_{b}^{-1} \mathcal{B}^{\bullet} \stackrel{+1}{\longrightarrow}
$$

Comme $j$ et $j_{b}$ sont affines, $R j_{*}$ et $R j_{b *}$ sont t-exacts. Les foncteurs $j_{b}^{-1}$ et ${ }^{p} \varphi_{\bar{f}-b}$ sont aussi t-exacts. On en déduit que $\mathcal{B}^{\bullet}$ ainsi que $R j_{b *} j_{b}^{-1} \mathcal{B}^{\bullet}$ sont des faisceaux pervers. Le théorème d'Artin nous permet de dire que $\mathbb{H}^{q}\left(\Sigma_{b}^{\infty}, i_{b}^{!} \mathcal{B}^{\bullet}\right)=0$ pour $q<-d(\bar{f})$ et $q>d(\bar{f})+1$.

Dans le cas de la dernière affirmation, il suffit de remarquer que l'hypothèse implique que $i_{b}^{!} \mathcal{B} \bullet$ est un faisceau pervers.

\section{Théorèmes des cycles invariants}

Pour une application sympa $f: X \longrightarrow S$, on a un théorème de cycles invariants qui généralise les résultats similaires de [1] et de [19].

Théorème 4.1. (des cycles invariants locaux) Soit $f: X \longrightarrow S$ une application sympa. Soit $b \in B$ et soit $m_{b}^{k}: H^{k}(F) \longrightarrow H^{k}(F)$ l'opérateur de monodromie qui correspond à un lacet élémentaire dans $S$ qui tourne une fois autour de $b$.

Alors on a pour tout $q$

$\operatorname{dim} \operatorname{Ker}\left(m_{b}^{q-1}-1\right)+\operatorname{dim} \operatorname{Ker}\left(m_{b}^{q}-1\right)=b_{q}(F)-\operatorname{dim} V^{q}(b)+b_{2 n-q-1}^{c}\left(F_{b}\right)$

où $b_{p}^{c}\left(F_{b}\right)=\operatorname{dim} H_{c}^{p}\left(F_{b}\right)$ pour tout entier $p$.

Démonstration. Soit $g: T\left(F_{b}\right)^{*} \longrightarrow D_{b}^{*}$ la fibration localement triviale induite par $f$ au voisinage de la fibre $F_{b}$, où $T\left(F_{b}\right)^{*}=T\left(F_{b}\right) \backslash F_{b}$. La suite spectrale de Leray dégénère au terme $E_{2}$ et nous donne pour tout $q$

$$
\operatorname{dim} H^{0}\left(D_{b}^{*}, R^{q} g_{*} \mathbb{C}_{X}\right)+\operatorname{dim} H^{1}\left(D_{b}^{*}, R^{q-1} g_{*} \mathbb{C}_{X}\right)=\operatorname{dim} H^{q}\left(T\left(F_{b}\right)^{*}\right) .
$$

On a les égalités suivantes :

(i) $\operatorname{dim} H^{0}\left(D_{b}^{*}, R^{q} g_{*} \mathbb{C}_{X}\right)=\operatorname{dim} \operatorname{Ker}\left(m_{b}^{q}-1\right)$, 
(ii)

$$
\begin{aligned}
\operatorname{dim} H^{1}\left(D_{b}^{*}, R^{q-1} g_{*} \mathbb{C}_{X}\right)= & \left.-\chi\left(D_{b}^{*}, R^{q-1} g_{*} \mathbb{C}_{X}\right)\right) \\
& +\operatorname{dim} H^{0}\left(D_{b}^{*}, R^{q-1} g_{*} \mathbb{C}_{X}\right) \\
= & \operatorname{dim} \operatorname{Ker}\left(m_{b}^{q-1}-1\right),
\end{aligned}
$$

$\operatorname{car} \chi\left(D_{b}^{*}, R^{q-1} g_{*} \mathbb{C}_{X}\right)=\chi\left(D_{b}^{*}\right) b_{q-1}(F)=0$.

D'autre part pour calculer $b_{q}\left(T\left(F_{b}\right)^{*}\right)$ on utilise la dualité de Poincaré

$$
b_{q}\left(T\left(F_{b}\right)^{*}\right)=b_{2 n-q}^{c}\left(T\left(F_{b}\right)^{*}\right)
$$

et la suite exacte

$$
\cdots \rightarrow H_{c}^{2 n-q-1}\left(F_{b}\right) \rightarrow H_{c}^{2 n-q}\left(T\left(F_{b}\right)^{*}\right) \stackrel{\alpha^{2 n-q}}{\longrightarrow} H_{c}^{2 n-q}\left(T\left(F_{b}\right)\right) \rightarrow H_{c}^{2 n-q}\left(F_{b}\right) \rightarrow \cdots
$$

Le morphisme $\alpha^{2 n-q}$ est le dual du morphisme induit par l'inclusion

$$
i_{b}^{q}: H^{q}\left(T\left(F_{b}\right)\right) \longrightarrow H^{q}\left(T\left(F_{b}\right)^{*}\right) .
$$

L'inclusion $F_{b^{\prime}} \longrightarrow T\left(F_{b}\right)$ se factorise comme

$$
F_{b^{\prime}} \longrightarrow T\left(F_{b}\right)^{*} \longrightarrow T\left(F_{b}\right)
$$

et cela, plus la condition que $f$ soit sympa, nous donne que le morphisme $\alpha^{2 n-q}$ est surjectif et donc l'égalité.

(iii) $b_{2 n-q}^{c}\left(T\left(F_{b}\right)^{*}\right)=b_{2 n-q}^{c}\left(T\left(F_{b}\right)\right)+b_{2 n-q-1}^{c}\left(F_{b}\right)$.

Finalement, on a $b_{2 n-q}^{c}\left(T\left(F_{b}\right)\right)=b_{q}\left(T\left(F_{b}\right)\right)=b_{q}(F)-\operatorname{dim} V^{q}(b)$.

On peut suivre la même idée de démonstration et obtenir le résultat suivant, qui généralise aussi certains résultats de [1] et de [19].

Théorème 4.2. (des cycles invariants globaux) Soit $f: X \longrightarrow S$ une application régulière non constante. On pose $S^{*}=S \backslash B, X^{*}=f^{-1}\left(S^{*}\right)$. Pour tout $k$, on dénote par $H^{k}(F)^{\mathrm{inv}}$ la partie invariante de la cohomologie par rapport à l'action de la monodromie du groupe fondamental $\pi_{1}\left(S^{*}\right)$.

Alors, on a pour tout $q$

$\operatorname{dim} H^{q-1}(F)^{\mathrm{inv}}+\operatorname{dim} H^{q}(F)^{\mathrm{inv}}=$

$$
=(\chi(S)-|B|) b_{q-1}(F)+\sum_{b \in B} b_{2 n-q-1}^{c}\left(F_{b}\right)+b_{q}(X)-\operatorname{dim} \operatorname{Ker} \beta^{q}-\operatorname{dim} \operatorname{Ker} \beta^{q+1}
$$

où $\beta^{k}: H^{k}(X) \longrightarrow H^{k}\left(X^{*}\right)$ est le morphisme induit par l'inclusion $X^{*} \longrightarrow X$. 


\section{Exemples}

5.1. Cas $d(\bar{f})=0$ ou 1

Remarque 5.1. Si $n=\operatorname{dim} X$ et si $d(\bar{f})=0$ alors $V^{q}(b)=0$ pour tout $q \leq n-2$ et tout $b$. Donc

$$
R^{q} f_{*} \mathbb{C}_{X}=\widetilde{H^{q}(F)} \text { pour } q \leq n-2
$$

et

$$
R^{n-1} f_{*} \mathbb{C}_{X}={ }^{p} R^{n} f_{*} \mathbb{C}_{X}[-1]=D R_{S}\left(\mathcal{G}_{f}^{0}\right)[-1]
$$

où $\mathcal{G}_{f}^{0}=\mathcal{H}^{0} f_{+}\left(\mathcal{O}_{X}\right)$ est le sytème de Gauss-Manin de $f$ en degré 0 , voir [21], [10]. Le faisceau constructible $R^{n-1} f_{*} \mathbb{C}_{X}$ peut donc être calculé à partir d'un D-module régulier holonome.

Remarque 5.2. Supposons que $d(\bar{f})=0$ et $S=\mathbb{C}$. Alors le morphisme induit par l'inclusion $i^{q}: H^{q}(X) \longrightarrow H^{q}(F)$ est un isomorphisme pour $q \neq n-1, n$ et on a la suite exacte

$$
0 \longrightarrow H^{n-1}(X) \longrightarrow H^{n-1}(F) \longrightarrow H^{n}(X, F) \longrightarrow H^{n}(X) \longrightarrow H^{n}(F) \longrightarrow 0 .
$$

Si on écrit le théorème 4.2 pour $q=n-1$ on obtient l'égalité :

$$
\operatorname{dim} H^{n-1}(F)^{\mathrm{inv}}=\sum_{b \in B} b_{n}^{c}\left(F_{b}\right)-|B| b_{n-2}(X)+b_{n-1}(X)+\operatorname{dim}\left(\operatorname{Ker} \beta_{n}\right) .
$$

En effet, on a $H^{n-2}(F)^{\text {inv }}=H^{n-2}(F)$ car $i^{n-2}$ est un isomorphisme. On a aussi Ker $\beta_{n-1}=0$ car $i^{n-1}$ est un monomorphisme et on a une factorisation $F \longrightarrow$ $X^{*} \longrightarrow X$.

Remarque 5.3. Lorsque $f: X \longrightarrow S$ est à singularités isolées sur $X$, on sait que l'on a

$$
\chi(S) \chi(F)=\chi(X)+(-1)^{n-1}(\mu+\lambda)
$$

où $\mu$ est le nombre de Milnor associé à $f$ et

$$
\lambda=\sum_{b \in B} \chi\left(\Sigma_{b}^{\infty},{ }^{p} \varphi_{t_{b} \circ \bar{f}}\left(R j_{*} \mathbb{C}_{X}[n]\right)\right) .
$$

Cette égalité nous permet de retrouver les résultats de [2], [5] (où ils montrent comment le calculer) ainsi que ceux de [22], [23] et [13]. On peut préciser la formule nous donnant $\lambda$ lorsque $d(\bar{f})=0$ ou 1 , et $j$ affine.

(i) $\operatorname{Cas} d(\bar{f})=0$. On a

$$
\lambda=\sum_{b \in B} \sum_{x \in \Sigma_{b}^{\infty}} \operatorname{dim} \mathcal{H}^{0}\left({ }^{p} \varphi_{\bar{f}-b}\left(R j_{*} \mathbb{C}_{X}[n]\right)\right)_{x},
$$

en particulier $\lambda$ est positif ou nul. 
(ii) Cas $d(\bar{f})=1$. On a

$$
\lambda=\sum_{b \in B} \sum_{x \in \Sigma_{b}^{\infty}} \operatorname{dim} \mathcal{H}^{0}\left({ }^{p} \varphi_{\bar{f}-b}\left(R j_{*} \mathbb{C}_{X}[n]\right)\right)_{x}-\sum_{b \in B} \chi\left(\Sigma_{b}^{\infty}, \mathcal{H}^{-1}\left({ }^{p} \varphi_{\bar{f}-b}\left(R j_{*} \mathbb{C}_{X}[n]\right)\right)\right) .
$$

On va voir plus loin que l'on peut avoir $\lambda \leq 0$.

Maintenant, on va considérer un exemple où l'on calcule les cycles évanescents de deux façons différentes. On va montrer que la condition d'avoir un plongement $j$ affine peut «transformer»des cycles évanescents à support fini en des cycles évanescents à support non fini.

\subsection{Cas $l: \mathbb{C}^{3} \backslash C \longrightarrow \mathbb{C}$ où $C$ est une courbe lisse intersection complète et $l$ une forme linéaire générique}

On considère l'espace projectif $\mathbb{P}^{3}$ avec pour coordonnées $[x, y, z, u]$.

Soit $Z: F(x, y, z, u)=0, \quad G(x, y, z, u)=0$ une courbe lisse, intersection complète de degré $(d, e)$, c'est-à-dire $d=\operatorname{deg}(F)$, et $e=\operatorname{deg}(G)$. On suppose que le pinceau d'hyperplans $\lambda x+\mu y=0$ est un pinceau de Lefschetz par rapport à $Z$ et que $\{u=0\}$ est transverse à $Z$.

Alors, soit $\mathbb{C}^{3}=\mathbb{P}^{3} \backslash\{u=0\}$ avec pour coordonnées $(x, y, z), C=Z \cap \mathbb{C}^{3}, l: \mathbb{C}^{3} \rightarrow$ $\mathbb{C}, l(x, y, z)=x$.

Avec ces conditions, on a

(i) $l_{\mid C}$ est propre et il y a exactement $c$ points critiques $a_{1}, \cdots, a_{c}$ pour $l_{\mid C}: C \rightarrow \mathbb{C}$, tous de multiplicité 2 , où $c=\operatorname{classe}(Z)$ (on a $c=2 d e-\chi(Z)[16]$, p. 25, $\chi(Z)=(4-d-e) d e[7]$, p. 152). En outre $l\left(a_{i}\right) \neq l\left(a_{j}\right)$ pour $i \neq j$.

(ii) Si $j_{0}: \mathbb{C}^{3}=\mathbb{C} \times \mathbb{C}^{2} \rightarrow \mathbb{C} \times \mathbb{P}^{2}$ est l'inclusion naturelle, alors $j_{0}(C)$ est fermé dans $\mathbb{C} \times \mathbb{P}^{2}$ (sinon on a une contradiction avec $l_{\mid C}$ propre).

On considère $X=\mathbb{C}^{3} \backslash C, \bar{X}=\mathbb{C} \times \mathbb{P}^{2}, L_{\infty}=\mathbb{P}^{2} \backslash \mathbb{C}^{2}$ la droite à l'infini et la stratification (de Whitney) de $\bar{X}$ donnée par

$$
\mathcal{S}_{\bar{X}} \quad: \quad X \coprod C \coprod \mathbb{C} \times L_{\infty}
$$

On a $\operatorname{Sing}\left(\bar{l}_{\mid \mathbb{C}^{3} \backslash C}\right)=\operatorname{Sing}\left(\bar{l}_{\mid \mathbb{C} \times L_{\infty}}\right)=\emptyset$ et $\operatorname{Sing}\left(\bar{l}_{\mid C}\right)=\left\{a_{1}, \ldots a_{c}\right\}=A$ où $\bar{l}: \mathbb{C} \times \mathbb{P}^{2}=$ $\bar{X} \rightarrow \mathbb{C}$ est la projection sur le premier facteur.

On va aussi noter $j=j_{0 \mid \mathbb{C}^{3} \backslash C}$ la restriction de l'injection $j_{0}$ à $\mathbb{C}^{3} \backslash C$.

Le faisceau $\mathcal{F}^{\bullet}=R j_{*}\left(\mathbb{C}_{X}\right)$ est un faisceau $\mathcal{S}_{\bar{X}}$-constructible car on a le résultat suivant

Proposition 5.1. Soient $X$ un espace muni d'une stratification de Whitney $\mathcal{S}_{X}, S \in$ $\mathcal{S}_{X}$ une strate et $j: S \hookrightarrow X$ l'inclusion. Alors $\mathcal{F}^{\bullet}=R j_{*} \mathbb{C}_{S}$ est cohomologiquement constructible par rapport à $\mathcal{S}_{X}$. 
Démonstration. Il suffit d'utiliser la trivialité topologique le long d'une strate $T$, $T \in \bar{S}$.

- Calcul de $\mathcal{H}^{q}\left(\varphi_{\bar{l}-s}\left(\mathcal{F}^{\bullet}\right)\right)_{a}$ pour $a \in\left(\{s\} \times \mathbb{P}^{2}\right) \cap A$.

Soit $a \in\left(\{s\} \times \mathbb{P}^{2}\right) \cap A, F_{a}:=\bar{l}^{-1}(\beta) \cap B_{\epsilon}(a)$ la fibre de Milnor de $\bar{l}$ en $a$ où $B_{\epsilon}(a)$ est la boule ouverte de centre $a$ est de rayon $\epsilon$, pour $\epsilon>0$ assez petit et pour un certain plongement local de $X$ dans un espace affine et $\beta$ suffisamment proche de $\bar{l}(a)$.

On a

$$
\mathcal{H}^{q}\left(\varphi_{\bar{l}-s}\left(\mathcal{F}^{\bullet}\right)\right)_{a}=\mathbb{H}^{q+1}\left(B_{\epsilon}(a), F_{a}, \mathcal{F}^{\bullet}\right)=H^{q+1}\left(B_{\epsilon}(a) \backslash C, F_{a} \backslash C, \mathbb{C}\right) .
$$

Une étude du couple $\left(B_{\epsilon}(a) \backslash C, F_{a} \backslash C\right)$ nous dit qu'il est homotopiquement équivalent au couple $\left(\left(S^{3} \times[0,1]\right) / \sim, S^{3} \times\{0\} \vee_{p_{0}} S^{3} \times\{1\}\right)$ où $(x, s) \sim(y, t)$ si $x=y=p_{0}$ est un point fixé de $S^{3}$.

D'où le résultat

$$
\varphi_{\bar{l}-s}^{q}\left(\mathcal{F}^{\bullet}\right)_{a}=\left\{\begin{array}{l}
\mathbb{C} \text { si } q=3 \text { et } a \in\left(\{s\} \times \mathbb{P}^{2}\right) \cap A, \\
0 \text { sinon. }
\end{array}\right.
$$

L'inclusion $j$ n'étant pas un morphisme affine, on peut aussi calculer ces cycles évanescents en passant par une autre compactification de $X$, qui a un plongement $j_{1}$ affine.

Soit $\bar{X}_{1}$ l'éclatement de $\bar{X}$ le long de $Z \cap \bar{X}, \pi: \bar{X}_{1} \rightarrow \bar{X}$ le morphisme naturel et $E=\pi^{-1}(Z \cap \bar{X})$. On note aussi $j_{1}: X \hookrightarrow \bar{X}_{1}$ l'injection naturelle et $\bar{l}_{1}=\bar{l} \circ \pi$.

On considère la stratification (de Whitney) de $\bar{X}_{1}$ donnée par

$$
\mathcal{S}_{\bar{X}_{1}} \quad: \quad X \coprod E \coprod \mathbb{C} \times L_{\infty}
$$

On a $\operatorname{Sing}_{\mathcal{S}_{\bar{X}_{1}}}\left(\bar{l}_{1}\right)=A \times \mathbb{P}^{1}$ et $\operatorname{Sing} \bar{X}_{1}\left(\bar{l}_{1}\right)=\left\{\left(a_{1}, t_{a_{1}}\right), \ldots\left(a_{c}, t_{a_{c}}\right)\right\}$ où $t_{a_{i}}$ est un point de $\pi^{-1}\left(a_{i}\right) \simeq \mathbb{P}^{1}$.

En un point de $A$, on se ramène localement à la situation

$$
C: z=0, \quad y-x^{2}=0 \quad \text { et } \quad \bar{l}_{1}(x, y, z) \mapsto y .
$$

On a deux cartes et dans une des deux cartes $\bar{l}_{1}$ s'écrit $(x, y, z) \mapsto x^{2}+y z$ et $E: y=0$.

La proposition 5.1 nous dit que le complexe de faisceaux $\mathcal{F}_{1}^{\bullet}:=R j_{1 *} \mathbb{C}_{X}$ est $\mathcal{S}_{\bar{X}_{1}}{ }^{-}$ constructible.

- Calcul de $\mathcal{H}^{q}\left(\varphi_{\bar{l}_{1}-s}\left(\mathcal{F}_{1}^{\bullet}\right)\right)_{(a, t)}$, pour $(a, t) \in \pi^{-1}\left(\left(\{s\} \times \mathbb{P}^{2}\right) \cap A\right)$.

Soit $(a, t) \in \pi^{-1}\left(\left(\{s\} \times \mathbb{P}^{2}\right) \cap A, F_{(a, t)}^{1}\right.$ la fibre de Milnor de $\bar{l}_{1}:=\bar{l}_{1}^{-1}(\beta) \cap B_{\epsilon}(a, t)$ en $(a, t), 0<\left|\bar{l}_{1}(a, t)-\beta\right| \ll \epsilon \ll 1$. 
On a

$$
\mathcal{H}^{q}\left(\varphi_{\bar{l}_{1}-s}\left(\mathcal{F}_{1}^{\bullet}\right)\right)_{(a, t)}=\mathbb{H}^{q+1}\left(B_{\epsilon}(a, t), F_{(a, t)}^{1}, \mathcal{F}_{1}^{\bullet}\right)=H^{q+1}\left(B_{\epsilon}(a, t) \backslash E, F_{(a, t)}^{1} \backslash E, \mathbb{C}\right) .
$$

Pour étudier le couple $\left(B_{\epsilon}(a, t) \backslash E, F_{(a, t)}^{1} \backslash E\right)$, on doit distinguer deux cas.

Premier cas $t \neq t_{a} .\left(B_{\epsilon}(a, t) \backslash E, F_{(a, t)}^{1} \backslash E\right)$ est homotope à $\left(\left(S^{1} \times[0,1]\right) / \sim, S^{1} \times\right.$ $\left.\{0\} \vee_{p_{0}} S^{1} \times\{1\}\right)$ où $(x, s) \sim(y, t)$ si $x=y=p_{0}$ est un point fixé de $S^{1}$.

D'où le résultat pour $t \neq t_{a}$

$$
\varphi_{\bar{l}_{1}-s}^{q}\left(\mathcal{F}_{1}^{\bullet}\right)_{(a, t)}=\left\{\begin{array}{l}
\mathbb{C} \text { si } q=1 \text { et }(a, t) \in \pi^{-1}\left(\left(\{s\} \times \mathbb{P}^{2}\right) \cap A\right) \\
0 \text { sinon. }
\end{array}\right.
$$

Deuxième cas $t=t_{a}$. On se ramène au germe d'hypersurface $x^{2}+y z=0$ et on utilise le difféomorphisme donné par [16], p. 37.

Le couple $\left(B_{\epsilon}\left(a, t_{a}\right) \backslash E, F_{\left(a, t_{a}\right)}^{1} \backslash E\right)$ est alors homotope à $\left(S^{1}, S^{1}\right)$ ce qui veut dire qu'il n'y a pas de cycles évanescents en ce point.

D'où le résultat

$$
\varphi_{\bar{l}_{1}-s}^{q}\left(\mathcal{F}_{1}^{\bullet}\right)_{(a, t)}=\left\{\begin{array}{l}
\mathbb{C} \text { si } q=1 \text { et }(a, t) \in \pi^{-1}\left(\left(\{s\} \times \mathbb{P}^{2}\right) \cap A\right), \quad t \neq t_{a}, \\
0 \text { sinon. }
\end{array}\right.
$$

L'avantage de la première compactification est que $\operatorname{dim} \operatorname{supp} \varphi_{\bar{l}-s}\left(\mathcal{F}^{\bullet}\right)=0$, son défaut est que le plongement $j$ n'est pas affine, en particulier le faisceau $R j_{*}\left(\mathbb{C}_{X}[3]\right)$ n'est pas pervers. La deuxième compactification corrige ce défaut mais par contre cette fois $\operatorname{dim} \operatorname{supp} \varphi_{\bar{l}_{1}-s}\left(\mathcal{F}_{1}^{\bullet}\right)=1$.

Remarque 5.4. Pour le cas $l: \mathbb{C}^{3} \backslash C \longrightarrow \mathbb{C}$ que l'on vient de discuter on peut calculer $b_{4}(X)$ et $b_{4}(F)$ et vérifier que $b_{4}(X) \neq b_{4}(F)$. Cela entraîne, via le théorème 3.1 , que $d(\bar{f})>0$ pour toute compactification avec $j$ affine.

Pour calculer $b_{4}(X)$ on utilise la suite exacte de Gysin pour une intersection complète lisse

$$
H^{4}\left(\mathbb{C}^{3}\right) \longrightarrow H^{4}(X) \stackrel{R}{\longrightarrow} H^{1}(C) \longrightarrow H^{5}\left(\mathbb{C}^{3}\right) \longrightarrow
$$

d'où $b_{4}(X)=b_{1}(C)=1-\chi(C)=1-(\chi(Z)-d e)=1+d e-(4-d-e) d e=$ $1-(3-d-e) d e \neq 0$. D'autre part $F=\mathbb{C}^{2} \backslash\{$ de points $\}$ a le type d'homotopie d'un bouquet de sphères de dimension 3 , donc $b_{4}(F)=0$. Des calculs de ce type nous permettent aussi de montrer que les applications $l$ et $\bar{l}_{1}$ sont des applications sympa.

Cet exemple est un cas particulier de la remarque 5.3 (i) et (ii). Ici, on peut calculer de deux façons différentes $\chi(F)$ ce qui va nous permettre de calculer le nombre $\lambda$. En effet on a

$$
\begin{aligned}
\chi(F) & =1-d e, \\
\chi(F)=\chi(X)+(\mu+\lambda) & =(1+d e-\chi(Z))+(\mu+\lambda), \\
\mu & =0 .
\end{aligned}
$$


On en conclut que $\lambda=-c$ est dans ce cas strictement négatif.

Si $H$ est un hyperplan générique de $\mathbb{C}^{3}$ alors on peut considérer $\bar{l}_{H}=\bar{l}_{\mid H \backslash C}$, $\lambda_{H}=\lambda\left(\bar{l}_{H}\right)$. On a $\chi\left(F_{\bar{l}_{H}}\right)=\chi(H \backslash C)-\lambda_{H}$ ce qui nous donne $\lambda_{H}=-d e$. Donc $\lambda+\lambda_{H}$ est strictement négatif contrairement au cas des polynômes à singularités isolées, voir [5], p. 133.

\section{Références}

[1] E. Artal-Bartolo, P. Cassou-Noguès, A. Dimca, Sur la topologie des polynômes complexes, Progress in Math. 162, Birhäuser 1998, 317-343.

[2] E. Artal-Bartolo, I. Luengo-Velasco, A. Melle-Hernandez, Milnor number at infinity, topology and Newton boundary of a polynomial function, Math. Z. 233 (2000), 679-696.

[3] A. Borel, N. Spaltenstein, Sheaf theoretic intersection cohomology, Intersection cohomology (Bern, 1983), Birkhäuser Boston, Boston, MA, 1984, pp. 47-182.

[4] S. A. Broughton, Milnor numbers and the topology of polynomial hypersurfaces, Invent. Math. 92 (1988), 217-241.

[5] P. Cassou-Noguès, A. Dimca, Topology of complex polynomials via polar curves, Kodai Math. J. 22 (1999), 131-139.

[6] P. Deligne, Le formalisme des cycles évanescents, dans SGA 7 XIII et XIV, Lect. Notes in Math. vol. 340, Springer, Berlin, 1973, pp. 82-115 et 116-164.

[7] A. Dimca, Singularities and topology of hypersurfaces, Springer-Verlag, New York, 1992.

[8] A. Dimca, Une introduction aux faisceaux pervers, notes d'un cours post DEA à l'université de Bordeaux 1, 1999, Notes rédigées par T. Brélivet. http ://www.math.u-bordeaux.fr/ brelivet/pervers.ps.gz

[9] A. Dimca, M. Saito, On the cohomology of the general fiber of a polynomial map, Compositio Math. 85 (1993), 299-309.

[10] A. Dimca, M. Saito, Algebraic Gauss-Manin systems and Brieskorn modules, Amer. J. Math. 123 (2001), 163-184.

[11] A. Dimca, A. Némethi, On the monodromy of complex polynomials, Duke Math. J. 108 (2001), 199-209.

[12] W. Fulton, Introduction to Toric Varieties, Annals of Math, Studies 131, Princeton University Press, 1993.

[13] S. M. Gusein-Zade, I. Luengo, A. Melle-Hernndez, Bifurcations and topology of meromorphic germs, New developments in singularity theory (Cambridge, 2000), 279-304, NATO Sci. Ser. II Math. Phys. Chem., 21, Kluwer Acad. Publ., Dordrecht, 2001.

[14] H. Hamm, On the cohomology of fibres, Trends in Singularities, eds. A. Libgober and M. Tibăr, Birkhäuser 2002.

[15] M. Kashiwara, P. Schapira, Sheaves on manifolds, Grund der Math. Wiss. 292, Springer Verlag 1990.

[16] K. Lamotke, The topology of complex projective varieties after S. Lefschetz, Topology 20 (1981), no. $1,15-51$. 
[17] Lê Dung Tráng, Sur les cycles évanouissants des espaces analytiques, C. R. Acad. Sci. Paris Sér. A-B 288 (1979), no. 4, A283-A285.

[18] F. Michel, C. Weber, On the monodromies of a polynomial map from $\mathbb{C}^{2}$ to $\mathbb{C}$, Topology 40 (2001), no. 6, 1217-1240.

[19] W. D. Neumann, P. Norbury, Vanishing cycles and monodromy of complex polynomials, Duke Math. J. 101 (2000), 487-497.

[20] C. Sabbah, On the comparaison theorem for elementary irregular D-modules, Nagoya Math. J., Vol. 141 (1996), 107-124.

[21] C. Sabbah, Hypergeometric periods for a tame polynomial, C. R. Acad. Sci. Paris Sér. I Math. 328 (1999), no. 7, 603-608 et preprint 1996.

[22] D. Siersma, M. Tibăr, Singularities at infinity and their vanishing cycles, Duke Math. J. Vol. 80 (1995), no. 3, 771-783.

[23] D. Siersma M. Tibăr, Vanishing cycles and singularities of meromorphic functions, preprint 1999. http ://www.math.uu.nl/people/siersma/mero30.ps.gz

[24] M. Tibăr, Topology at infinity of polynomial maps and Thom regularity condition, Compositio Math. 111 (1998), 89-109. 